\title{
Conhecimento do Enfermeiro da Atenção Primária à Saúde Sobre os Cuidados com o Pé Diabético
}

\author{
Knowledge of Nurses in Primary Health Care on the Care for the Diabetic Foot \\ Conocimiento del Enfermero de la Atención Primaria de Salud \\ Acerca de la Atención al Pie Diabético
}

\author{
Luciana Catunda Gomes de Menezes ${ }^{1}$, Maria Vilani Cavalcante Guedes ${ }^{1}$, Nádya dos Santos Moura', \\ Denizielle de Jesus Moreira Moura ${ }^{1,2}$, Luara Abreu Vieira', Ariane Alves Barros ${ }^{1}$
}

\begin{abstract}
RESUMO
O pé em risco é uma das complicações microvasculares graves e mutilantes da pessoa com diabetes e seu principal tratamento é a detecção precoce. Mediante conhecimento das causas do pé diabético, pacientes de alto risco poderão ser identificados precocemente, evitando amputações. Objetivou-se descrever o conhecimento do enfermeiro da Atenção Primária à Saúde sobre os cuidados prestados aos diabéticos com pé em risco, durante consulta de enfermagem. Estudo descritivo com dez enfermeiras de Unidades de Atenção Primária à Saúde de Fortaleza, Ceará, com dados coletados em entrevista semiestruturada e observação da consulta de enfermagem, realizadas em setembro e outubro de 2013. Projeto aprovado pelo Comitê de Ética em Pesquisa da Universidade Estadual do Ceará, sob n 201.279. Dos discursos emergiram as temáticas: conhecimento do enfermeiro sobre os cuidados com o pé diabético em risco e fatores influenciadores no atendimento do enfermeiro. Os enfermeiros sabem da importância dos cuidados educativos, porém, desconhecem a importância da avaliação clínica. Falta de conhecimentos sobre avaliação clínica requer educação permanente, recursos materiais, equipamentos e estrutura física como fatores influenciadores dessa assistência. Conclui-se que, para cuidar desses pacientes, os enfermeiros devem ter seus conhecimentos atualizados e específicos e condições materiais para minimizar o surgimento de complicações advindas da ausência desses cuidados.
\end{abstract}

DESCRITORES: Estomaterapia. Enfermagem. Cuidados de enfermagem. Diabetes mellitus. Pé diabético.

\begin{abstract}
At-risk foot is one of the serious and crippling microvascular complications for people with diabetes; and the main treatment is early detection. By knowing the causes of diabetic foot, high-risk patients might be identified early, thus avoiding amputations. We aimed to describe the knowledge of nurses in Primary Health Care on the care offered for diabetic patients with at-risk foot, during nursing consultation. Descriptive study with ten nurses from Primary Health Care Units of Fortaleza, Ceará, Brazil, with data collected through semi-structured interviews and observation of the nursing consultation, conducted in September and October 2013. The Research Ethics Committee of the Universidade Estadual do Ceará, under number 201.279, approved the project. The following themes emerged from the speeches: knowledge of nurses on the care for the diabetic foot and influencing factors on nurses' assistance. Nurses know the importance of educational care, but are unaware of the importance of clinical evaluation. As influencing factors for such assistance, there was lack of knowledge on clinical evaluation requiring continuing education, material resources, equipment, and physical infrastructure. Therefore, we conclude that nurses, in order to care for these patients, should have updated and specific knowledge, besides material conditions to minimize the occurrence of complications from lack of care.
\end{abstract}

DESCRIPTORS: Stomatherapy. Nursing. Nursing care. Diabetes mellitus. Diabetic foot.

\footnotetext{
1Programa de Pós-graduação em Cuidados Clínicos em Enfermagem e Saúde da Universidade Estadual do Ceará (UECE) - Fortaleza (CE), Brasil.

${ }^{2}$ Faculdade Metropolitana da Grande Fortaleza - Fortaleza (CE), Brasil.

Endereço para correspondência: Rua Valdetário Mota, 1514, apartamento 701 - Papicu - CEP: 60175-740 - Fortaleza (CE), Brasil -

E-mail: lucianacatundagomes@yahoo.com.br

Artigo recebido em: 16/10/2015 - Aceito para publicação em: 21/06/2016
} 


\section{RESUMEN}

El pie en riesgo es una de las complicaciones microvasculares graves y incapacitantes de personas con diabetes; y su tratamiento principal es la detección temprana. A través del conocimiento de las causas del pie diabético, pacientes de alto riesgo podrán ser identificados tempranamente, evitando amputaciones. El objetivo fue describir el conocimiento del enfermero de la Atención Primaria de Salud sobre atención a pacientes diabéticos con pie en riesgo, en consulta de enfermería. Estudio descriptivo, con diez enfermeras de Unidades de Atención Primaria de Salud de Fortaleza, Ceará, Brasil, con datos recolectados en entrevista semiestructurada y observación de la consulta de enfermería, en septiembre y octubre de 2013. Proyecto aprobado por el Comité de Ética en Investigación de la Universidade Estadual do Ceará, según número 201.279. De los discursos, emergieron las temáticas: conocimiento del enfermero sobre atención con el pie diabético en riesgo y factores que influyen en la atención. Los enfermeros saben de la importancia de la atención educativa, pero desconocen la importancia de la evaluación clínica. Desconocimiento sobre evaluación clínica requiere educación permanente, recursos materiales, equipamientos e infraestructura física, factores que influyen en la atención. En conclusión, para cuidar de estos pacientes, los enfermeros deben tener conocimientos actualizados y específicos, y condiciones materiales para minimizar complicaciones por falta de atención.

PALABRAS CLAVE: Estomaterapia. Enfermería. Atención de enfermería. Diabetes mellitus. Pie diabético.

\section{INTRODUÇÃO}

O diabetes mellitus (DM) é considerado um dos problemas de saúde mais importantes da atualidade, em virtude das elevadas morbidade e mortalidade causadas por complicações que comprometem a qualidade de vida dos pacientes ${ }^{1}$. Uma das complicações é o pé diabético, caracterizado por lesões isoladas ou diversas que ocorrem nos pés das pessoas com DM, geralmente decorrentes de tríade composta por neuropatia, doença arterial periférica (DAP) e infecções².

De modo geral, os problemas relacionados aos pés são complicações comuns às pessoas com DM, com prevalência de 23 a 42\% para neuropatia, 9 a 23\% para doença vascular, 5 a $7 \%$ para ulcerações 2 . Calcula-se em $25 \%$ a incidência de tais ulcerações nas pessoas com DM ao longo da vida, com 85\% dessas lesões precedendo amputações ${ }^{3}$. No Brasil, segundo estimativas, verificam-se 40 mil amputações por $\mathrm{ano}^{4}$. As úlceras de pé, além de serem complicações comuns e sérias, também apresentam mortalidade significativa. Em comparação com não diabéticos, o pé diabético tem um risco de amputação 15 vezes maior, correspondendo a $70 \%$ das amputações não traumáticas 5 .

Conforme sabido, a principal medida no tratamento dessas lesões é a detecção precoce, mediante condutas relacionadas à educação específica para os pés, do paciente e de seus familiares, e avaliação dos fatores de riscos por parte da equipe multiprofissional ${ }^{6}$.

O envolvimento dos familiares é essencial pela colaboração na realização de ações que o paciente sozinho não é capaz de executar.
Mediante o conhecimento das causas do pé diabético, pacientes de alto risco poderão ser identificados precocemente, evitando amputações. Nesse contexto, avaliação e medidas de prevenção do grau de risco acrescidas do estímulo ao autocuidado, atendimento interdisciplinar e educação em saúde poderão reduzir a ocorrência de lesões em até $50 \% \%^{3,4,7}$.

Entre as ações de enfermagem, a consulta de enfermagem (CE) configura-se como ação cada vez mais adotada nos serviços, como estratégia de promoção da saúde no contexto do cuidado.

Atividade privativa do enfermeiro, a CE utiliza componentes do método científico para identificar situações de saúde/doença, prescrever e implementar medidas de enfermagem que contribuam para promoção, proteção da saúde, prevenção de adoecimentos, recuperação e reabilitação do indivíduo, da família e comunidade ${ }^{8}$.

A CE constitui fator decisivo na prevenção de complicações nos pés de pessoas com DM, pois permite ao enfermeiro a identificação de situações problemas, uso de raciocínio clínico, determinação de diagnósticos, resultados e intervenções, favorecendo o processo de cuidar e educar e incentivando o paciente a desempenhar o autocuidado. Dessa maneira, reforça a adesão ao tratamento clínico?.

Nesse contexto, por ser o profissional enfermeiro qualificado para a $\mathrm{CE}$, este estudo foi norteado pelas seguintes questões: quais as estratégias de avaliação de fatores de riscos em pés de pessoas diabéticas realizadas durante a CE? Qual o conhecimento dos enfermeiros no tocante à prevenção de complicações no pé do paciente diabético em risco? 


\section{OBJETIVO}

Descrever o conhecimento do enfermeiro da Atenção Primária à Saúde sobre os cuidados prestados aos diabéticos com pé em risco, durante $\mathrm{CE}$.

\section{MÉTODO}

Estudo descritivo, com ênfase nas características da população ou fenômeno investigado, identificando prováveis relações entre as variáveis ${ }^{10}$. Adotou-se a abordagem qualitativa por se basear na premissa de que os conhecimentos sobre os indivíduos só são possíveis com a descrição da experiência humana, tal como é vivida e tal como é definida pelos próprios atores. Nesta pesquisa, o local escolhido foram as Unidades de Atenção Primária à Saúde (UAPS) em Fortaleza, Ceará.

A coleta de dados ocorreu em cinco UAPS localizados nas proximidades do Campus da Universidade Estadual do Ceará (UECE), nos meses de setembro e outubro de 2013. Participaram dez enfermeiras escolhidas por acessibilidade e que atenderam aos critérios de inclusão: serem enfermeiras generalistas/ou estomaterapeutas e acompanharem pacientes com DM há pelo menos um ano nas unidades selecionadas para o estudo. Excluíram-se aquelas que estavam afastadas do serviço por qualquer motivo.

A coleta de dados ocorreu durante a consulta de enfermagem, por meio de entrevista semiestruturada e observação estruturada da avaliação clínica dos pés de pessoas com DM. Inicialmente, realizaram-se as entrevistas com as enfermeiras, coletaram-se os dados de identificação, formação profissional (tempo de formação, tempo de experiência com pessoas diabéticas, qualificação acadêmica, entre outros) e em seguida foram questionadas no que concerne ao conhecimento e à prática sobre avaliação clínica de pés de pessoas diabéticas por meio do exame físico.

Posteriormente, utilizou-se roteiro de observação. Com esse, os dados coletados previamente poderiam ser visualizados diretamente pela pesquisadora. Esse roteiro constou das seguintes informações: avaliação dos pés de pessoas com DM e atuação no processo de educar em saúde. Para garantir o anonimato das entrevistadas, elas foram codificadas pela letra "E", de entrevistadas, seguida de numeral arábico, conforme ordem das entrevistas. Em cumprimento ao exigido, a pesquisa foi aprovada pelo Comitê de Ética em Pesquisa da UECE, conforme parecer no 201.279. No desenvolvimento do estudo seguiram-se as recomendações da Resolução no 466/12, as participantes foram convidadas e aquelas que aceitaram assinaram o Termo de Consentimento Livre e Esclarecido. Também obteve-se permissão para realizar observação da consulta com a enfermeira.

Para a análise dos resultados, trabalhou-se com a técnica da análise de conteúdo, definida como um conjunto de técnicas de análise das comunicações que usa procedimentos sistemáticos e objetivos de descrição do conteúdo das mensagens em três fases: pré-análise; exploração do material; tratamento dos resultados, inferência e interpretação ${ }^{11}$. Passou-se, então, à leitura minuciosa dos depoimentos das enfermeiras, com vistas a apreender o significado do objeto de estudo e identificar as categorias que formaram as seguintes temáticas: conhecimento do enfermeiro sobre os cuidados preventivos com o pé diabético em risco e fatores influenciadores no atendimento do enfermeiro. Desse modo, procedeu-se à análise compreensiva dos resultados, cuja interpretação apoiou-se na literatura atualizada sobre pé diabético.

\section{RESULTADOS E DISCUSSÃO}

Participaram do estudo 10 enfermeiras, com idade entre 25 e 37 anos. Desempenhavam assistência ao paciente com DM nas UAPS investigadas a aproximadamente quatro anos, com tempo de formação profissional superior a seis anos. Tais dados retrataram o perfil de profissionais experientes na área de atuação.

Quanto à qualificação, nove enfermeiros possuíam especialização. Desses, seis eram especialistas em Saúde da Família ou Saúde Pública; dois em Estomaterapia; um em Educação em Saúde. Um enfermeiro não tinha especialização. Em relação aos treinamentos implementados para acompanhamento de pessoas com DM e pé em risco, dois entrevistados haviam realizado cursos na área, porquanto desempenhavam trabalho com esse tipo de paciente em outros serviços.

Os profissionais foram entrevistados nas UAPS onde prestavam atendimento às pessoas com DM. Dos participantes, oito afirmaram realizar somente orientações básicas, como cuidados com a alimentação, prática de exercícios físicos, uso adequado da insulina, entre outras. No tocante à avaliação clínica do pé, dois relataram desempenhar cuidados mais específicos, como testes 
de sensibilidade com monofilamento de Semmes-Weisntein $5.07 \mathrm{de} 10 \mathrm{~g}$, diapasão de $128 \mathrm{~Hz}$, avaliações dos pulsos periféricos, condições da pele, deformidades, alteração na biomecânica, presença de ulcerações e outros.

Com base na leitura do conteúdo foi possível definir as unidades de análise ou unidades de registro (partes da frase), as quais foram categorizadas por semelhança, e de acordo com os critérios semânticos, divididas em categorias temáticas, conforme exposto.

\section{Conhecimento do enfermeiro sobre os cuidados preventivos com o pé diabético em risco}

Essa temática reflete o conhecimento dos enfermeiros sobre os cuidados educativos e cuidados clínicos com o pé diabético em risco. Como evidenciado, o enfermeiro entende a gravidade, as consequências da doença e a necessidade de uma avaliação mais específica, porém, a maioria canaliza sua atenção apenas para a educação em saúde.

Aqui realmente a gente não presta muita atenção no pé do diabético. É uma falha nossa. Quando ele cita que tem algum problema é que a gente olha e encaminha. (E3)

Geralmente, a gente faz avaliação mais teórica, avaliação física do pé ninguém faz. (E4)

Faço muitas orientações, como: controle glicêmico, uso correto das insulinas, caso faça uso, prática de exercícios físicos, pois tendo sido realizado isso, o resto fica mais fácil. (E8)

Inegavelmente, a educação em saúde é parte fundamental no tratamento da pessoa com diabetes, porém, a princípio, tratar do pé em risco não abrange somente essas orientações indicadas pelas enfermeiras; requer também conhecimentos mais específicos sobre a avaliação clínica do pé. $\mathrm{O}$ enfermeiro desempenha atividades importantes nos diversos níveis de atenção à saúde, como agente cuidador e/ou educador, mas para que essas ações sejam de qualidade, são necessários conhecimento, habilidade e competência ${ }^{12}$.

O cuidado preventivo desenvolvido pela enfermagem ao paciente com pé diabético envolve vários níveis, desde a identificação do risco, por meio de exame clínico detalhado, até avaliação mais específica do pé, como investigação de neuropatia e verificação dos pulsos distais ${ }^{13}$.
Pequenos investimentos em prevenção e educação podem significar menos amputações, aumento na qualidade de vida e considerável redução nos custos para o sistema de saúde. Essa complicação vem preocupando profissionais do setor saúde, cuja prevenção se tornou desafio.

Neste âmbito, a educação também constitui ferramenta importante durante a consulta de enfermagem, porquanto 85\% dos problemas decorrentes do pé diabético são passíveis de prevenção, mediante cuidados especializados ${ }^{14}$.

Contudo, o planejamento de práticas educativas realizadas por enfermeiros deverá incluir medidas simples e específicas do pé em risco, como sapatos apropriados, higiene, hidratação, corte adequado das unhas, entre outras. Tais medidas contribuirão para a prevenção de úlceras, minimizando a influência dos riscos e o número de amputações. Reforça-se o uso de meias adequadas para evitar o surgimento de micoses interdigitais e onicomicoses, condições que agravam o pé diabético ${ }^{4}$.

É sabida a necessidade de conhecimento dos profissionais para realizar o acompanhamento desses pacientes, porém, o exame dos pés é quase sempre negligenciado, apesar das diretrizes e recomendações existentes. Diante disso, o consenso International Working Group on the Diabetic Foot (IWGDF) ${ }^{15}$ sugere cuidados em cinco estratégias: inspeção e exame regular dos pés; identificação do pé em risco; educação dos familiares, pacientes e profissionais; uso de calçado adequado; e tratamento da doença não ulcerativa. Em relação aos cuidados clínicos com o pé em risco, os profissionais se mostraram capacitados para a execução de exame clínico mais específico. $\mathrm{O}$ enfermeiro, como membro da equipe de saúde, é um elemento multiplicador de conhecimento por meio da promoção de educação em saúde e avaliação do grau de risco, contribuindo para o desenvolvimento de hábitos saudáveis que proporcionarão mais segurança e melhor aceitação da doença.

É relevante destacar depoimentos de enfermeiras quanto à avaliação da sensibilidade do pé. Dentre o grupo, coube enfatizar o relato de $\mathrm{E} 1$, quando mencionou as avaliações efetuadas em conformidade com as recomendações do consenso $\mathrm{IWGDF}^{15}$.

Realizo avaliação do pé anualmente e quando tem risco, avalio semestral e trimestralmente. Avaliação dermatológica, avaliação vascular, sensibilidade protetora, rastreamento da neuropatia e biomecânica do pé. Peço para caminhar, sem o calçado. Vejo se tem alteração na marcha, postural, deformidade óssea. Vejo se o calçado é adequado. (E1) 
A gente explica sobre os calçados, os exames diários dos pés ao deitar, secagem dos espaços interdigitais. Se houver qualquer lesão procurar imediatamente o Centro de Saúde.(E5)

A execução de adequado exame físico dos pés é indispensável para identificação precoce de sinais e sintomas passíveis de desencadear úlcera neuropática ${ }^{16}$. Aponta-se redução entre 44 e $85 \%$ com os cuidados preventivos, efetivos e específicos com os pés ${ }^{17}$.

Em todos os momentos, é fundamental manter a doença com o máximo de controle possível para o tratamento do pé em risco; mas, além de controle glicêmico eficaz verificado por meio dos testes glicêmicos, deve-se promover avaliação criteriosa e específica dos pés. Nessa avaliação, os seguintes pontos são essenciais: verificar e avaliar deformidades dos pés (pé em martelo ou dedos em garra, proeminências ósseas), pele seca, calosidades, veias dilatadas, sinais de isquemia, deformidades ou danos nas unhas, detecção de neuropatia por monofilamento de Semmes-Weinstein (10 g), diapasão de $128 \mathrm{~Hz}$, sensação tátil ou dolorosa com uso do pino ou palito, palpação de pulsos periféricos (pedioso e tibial posterior), tempo de enchimento venoso superior a 20 segundos e índice de tornozelo braquial (ITB) ${ }^{2}$.

Como observado, os depoimentos de enfermeiras estomaterapeutas sinalizaram conhecimento e preocupação em executar exame clínico eficaz, no intuito de evitar complicações decorrentes da doença. De modo geral,o estomaterapeuta é um profissional constantemente interessado nos avanços técnicos e científicos referentes aos cuidados com esses pacientes, os quais possibilitam avaliação clínica satisfatória do pé em risco. Todavia, nem sempre se encontram esses profissionais nos serviços.

\section{Fatores influenciadores no atendimento do enfermeiro}

Como mostra essa temática, apesar das dificuldades apresentadas, estas não inviabilizaram o propósito das enfermeiras em desempenhar com competência as atividades no cotidiano e em manter como meta a qualidade da assistência de enfermagem. Porém, a carência de recursos materiais, de equipamentos e até mesmo de ambiente foi apontada como fator que compromete o trabalho do enfermeiro, pois repercute no processo de trabalho e na satisfação do usuário com o serviço oferecido.

Têm muitas dificuldades. Não temos sala, estou nessa sala porque a médica não veio hoje pela manhã. (E3)
As dificuldades são muitas. Falta estrutura, não temos sala própria para fazer a avaliação, não temos insumos básicos para os curativos. (E4)

Falta material didático para orientar, um panfleto, falta estrutura para criar grupos específicos, falta material para realizar o curativo, só tem soro fisiológico. (E2)

Nas dificuldades, acho que o pior é a falta de recursos, às vezes falta o básico que são os remédios. (E6)

Às vezes preciso radiografar o pé de um paciente [...]. Outro dia, estava com um paciente com suspeita de osteomielite e não tinha nada. (E5)

As carências apontadas dificultavam a realização do trabalho dos enfermeiros investigados, sobretudo porque determinadas situações não dependiam da existência desses para um atendimento mais qualificado. Contudo, as situações associadas a outras necessidades identificadas podem tanto comprometer o sentimento do profissional em relação ao trabalho quanto sobrecarregá-lo por não vislumbrar mudanças. Constatou-se, também, desmotivação desses profissionais. Estudo realizado em Aracaju, Sergipe, sobre motivação da equipe de enfermagem evidenciou que $60 \%$ das pesquisadas se consideraram desmotivadas em seu ambiente de trabalho. Como principais fatores $\mathrm{da}$ desmotivação citaram-se os seguintes: sobrecarga de trabalho, falta de reconhecimento dos gestores, falta de um bom relacionamento entre a gerência de enfermagem e a equipe assistencial e falta de capacitação dos profissionais ${ }^{18}$. Para um desempenho profissional eficaz, é imprescindível a atualização contínua com vistas a acompanhar os avanços técnicos e científicos ${ }^{19}$.

Inegavelmente, a ausência de equipamentos, materiais e medicamentos essenciais para a prática profissional configura-se em um conjunto de problemas de ordem objetiva e subjetiva para o trabalhador: desorganização, interrupções constantes do trabalho, exposição a riscos diversos, tanto para a pessoa que está sendo cuidada quanto para o profissional, ansiedade e sensação de trabalho incompleto ${ }^{20}$. Em face dos depoimentos das enfermeiras, verificou-se, também, ausência de equipamentos e materiais específicos para a realização da avaliação clínica e de curativos.

A maior dificuldade é o tratamento tópico, de fato não temos nenhum produto, agora que chegou a pomada sulfadiazina de prata, só que eu preciso de um produto bom, pois eu digo 'gente não posso fazer milagre'. (E5) 
Dificuldades são muitas, mas principalmente de material necessário para uma avaliação completa e para realizar os curativos. (E7)

De modo geral, as UAPS cenários do estudo não dispõem de variedades de produtos, limitando-se a pomadas enzimáticas ou soro fisiológico. Entretanto, a complexidade de produtos de uso tópico no tratamento de feridas crônicas, incluindo aqueles que controlam a infecção, é fundamental para o alcance da cicatrização em menor tempo e com menor custo. A gravidade das complicações e os meios apropriados para controlá-las torna o DM uma doença onerosa, não apenas para os pacientes afetados e suas famílias, como também para o Sistema Único de Saúde (SUS) ${ }^{20}$.

Nos Estados Unidos da América, por exemplo, os gastos com esses pacientes são estimados em valores duas a três vezes superiores aos de um indivíduo sem a doença.

Diante dessas dificuldades relatadas, a presença de equipe composta por especialistas foi referida como importante na avaliação da pessoa com DM.

Temos dificuldades de encaminhamento para outros serviços. (E6)

Quando precisamos de um vascular (cirurgião), por exemplo, não temos, aí demora para encaminharmos, e o paciente vai complicando. (E9)

De acordo com as diretrizes do consenso IWGDF ${ }^{15}$, a consulta a esses pacientes deve englobar endocrinologistas, cirurgiões vasculares e ortopédicos, enfermeiras e um técnico de órteses. Deve contar, ainda, com o apoio indispensável de fisiatras ${ }^{16}$. Nas unidades pesquisadas, $\mathrm{o}$ atendimento era prestado por médicos e enfermeiros. Mesmo com essas dificuldades, a presença de médico na equipe precisa ser acessível e foi mencionada como significativa e facilitadora nesse processo de atendimento.

Nas facilidades, encontro a parceria com toda equipe, principalmente com o médico coordenador, pois me dá aval de tudo que preciso. (E5)

Como facilidades, temos acesso fácil aos médicos da nossa unidade e dos agentes de saúde que vão ao domicílio. (E6)
É oportuno destacar a necessidade de trabalho interdisciplinar, na perspectiva de envolvimento mais sólido de profissionais na prestação de assistência de melhor qualidade, demonstrando o potencial dessas experiências na mudança da prática clínica desses pacientes. O DM não requer tecnologia sofisticada para o diagnóstico e tratamento. Contudo, as medidas de prevenção são estratégias poderosas na redução da morbidade e da mortalidade.

\section{CONCLUSÃO}

O estudo conseguiu alcançar o objetivo proposto, demostrando haver um déficit de conhecimento dos enfermeiros generalistas na avaliação clínica do pé de diabético no seu cotidiano profissional. Os seus conhecimentos se baseavam numa assistência focada na troca de coberturas ou simplesmente em orientações básicas sobre a doença, como: cuidados com a alimentação, prática de exercícios físicos, uso correto de insulinas, dentre outras. Os principais motivos relatados para a não execução da avaliação clínica do pé estão relacionados, na verdade, com as condições inadequadas de trabalho; como: escassez de material, recursos físicos e educação permanente. Assim sendo, é importante que o enfermeiro generalista adquira conhecimento técnico e científico para a realização da avaliação clínica do pé diabético. Por sua vez, o enfermeiro estomaterapeuta, mediante um conhecimento mais especializado, foi o profissional mais habilitado para executar os cuidados específicos com o pé diabético, como apoio no uso de equipamentos como monofilamento, diapasão, palpação dos pulsos, orientações sobre calçado terapêutico, corte adequado das unhas, higiene, dentre outros.

Ressalta-se que este estudo poderá estimular os enfermeiros generalistas e os gestores da atenção primária em relação à assistência à pessoa com $\mathrm{DM}$ e pé em risco a encontrarem outras formas e técnicas de obtenção de conhecimentos, e as instituições a desenvolverem educação permanente, como possibilidade de prevenir ou retardar o desencadeamento de complicações agudas e crônicas. Desse modo, poderão ajudar na promoção da qualidade de vida desses pacientes. Assim, a pesquisa trouxe como contribuição importante a melhoria da assistência na consulta de enfermagem. Ao mesmo tempo, evidenciou algumas fragilidades: escassez de recursos materiais e estrutura física para realizar o atendimento e falta de educação permanente. 


\section{REFERÊNCIAS}

1. American Diabetes Association. Diagnosis and classification of diabetes mellitus. Diabetes Care. 2014;37(Suppl 1):S81-90.

2. Sociedade Brasileira de Diabetes. Diretrizes da Sociedade Brasileira de Diabetes. 5ª ed. São Paulo: AC Farmacêutica; 2014.

3. Duarte N, Gonçalves A. Pé diabético. Angiol Cir Vasc. 2011;7(2):65-79

4. Audi EG, Moreira RC, Moreira ACMG, Pinheiro EFC, Mantovani MF, Araújo AG. Avaliação dos pés e classificação do risco para pé diabético: contribuições da enfermagem. Cogitare Enferm. 2011;16(2):240-6.

5. Anselmo MI, Nery M, Parisi MCR. The effectiveness of educational practice in diabetic foot: a view from Brazil. Diabetol Metab Syndr. 2010;2(1):45.

6. Martin VT, Rodrigues CDS, Cesarino CB. Conhecimento do paciente com diabetes mellitus sobre o cuidado com os pés. Rev Enferm UERJ. 2011;19(4):621-5.

7. Tavares DMS, Dias FA, Araújo LR, Pereira GA. Perfil de clientes submetidos a amputações relacionadas ao diabetes mellitus. Rev Bras Enferm. 2009;62(6):825-30.

8. Conselho Federal de Enfermagem. Resolução COFEN n 358/2009. Dispõe sobre a Sistematização da Assistência de Enfermagem e a implementação do Processo de Enfermagem em ambientes, públicos ou privados, em que ocorre o cuidado profissional de Enfermagem, e dá outras providências. Brasília: Diário Oficial da União; 2009.

9. Silva SH, Cubas MR, Fedalto MA, Silva SR, Lima TCC. Estudo avaliativo da consulta de enfermagem na rede básica de Curitiba - PR. Rev Esc Enferm USP. 2010;44(1):68-75.

10. Gil AC. Como elaborar projetos de pesquisa. $5^{a}$ ed. São Paulo: Atlas; 2010

11. Bardin L. Análise de conteúdo. $2^{\mathrm{a}}$ reimpr. Lisboa: Edições 70; 2010
12. Benito GAV, Tristão KM, Paula ACSF, Santos MAS, Ataide LJ, Lima RCD. Desenvolvimento de competências gerais durante o estágio supervisionado. Rev Bras Enferm. 2012;65(1):172-8

13. Bakker K, Apelqvist J, Schaper NC. Practical guidelines on the management and prevention of the diabetic foot 2011. Diabetes Metab Res Rev. 2012;28(Suppl 1):225-31.

14. Rocha RM, Zanetti ML, Santos MA. Comportamento e conhecimento: fundamentos para prevenção do pé diabético. Acta Paul Enferm. 2009;22(1):17-23.

15. The International Working Group on the Diabetic Foot. International Consensus on the Diabetic Foot. 2011. DVD.

16. Moreira RC, Cruz CFR, Valsecchi EASS, Marcon SS. Vivências em família das necessidades de cuidados referentes à insulinoterapia e prevenção do pé diabético. Rev Gaúcha Enferm. 2008;29(2):283-91.

17. Bona SF, Barbosa MAR, Ferraz CLH, Guarita LKS, Nina RVAH, Barbosa NMRF, et al. Prevalência do pé diabético nos pacientes atendidos na emergência de um hospital público terciário de Fortaleza. Rev Bras Clin Med. 2010;8(1):1-5.

18. Bezerra FD, Andrade MFC, Andrade JS, Vieira MJ, Pimentel D. Motivação da equipe e políticas motivacionais adotadas pelo enfermeiro. Rev Bras Enferm. 2010;63(1):33-7.

19. Melo EM, Fernandes VS. Avaliação do conhecimento do enfermeiro acerca das coberturas de última geração. Rev Estima. 2011;9(4):12-20

20. David HMSL, Mauro MYC, Silva VG, Pinheiro MAS, Silva FH. Organização do trabalho de enfermagem na atenção básica: uma questão para a saúde do trabalhador. Texto Contexto Enferm. 2009;18(2):206-14. 